\title{
Effect of influenza vaccination on oxidative stress products in breath
}

\author{
Michael Phillips ${ }^{1,2,6}$, Renee N Cataneo ${ }^{1}$, Anirudh Chaturvedi ${ }^{1}$, \\ Patrick J Danaher $^{3}$, Anantrai Devadiga ${ }^{1}$, David A Legendre ${ }^{4}$, \\ Kim L Nail ${ }^{4}$, Peter Schmitt ${ }^{5}$ and James Wai ${ }^{1}$ \\ ${ }^{1}$ Menssana Research Inc., Fort Lee, NJ 07024-6510, USA \\ ${ }^{2}$ Department of Medicine, New York Medical College, Valhalla, NY, USA \\ ${ }^{3}$ Eglin USAF Regional Hospital, 96 MDG/96 MDOS/SGOMI, Eglin AFB, FL 32542-1282, USA \\ ${ }^{4}$ HQ AFSFC, 1517 Billy Mitchell Blvd, Bldg 954, Lackland AFB, TX 78236, USA \\ ${ }^{5}$ Schmitt \& Associates, 211 Warren St, Newark, NJ 07103, USA \\ E-mail: mphillips@menssanaresearch.com,rcataneo@menssanaresearch.com, \\ achaturvedi@menssanaresearch.com, adevadiga@menssanaresearch.com,jwai2006@gmail.com, \\ patrick.danaher@eglin.af.mil, David.Legendre@LACKLAND.AF.MIL, \\ Kim.Nail@LACKLAND.AF.MIL and peter.schmitt@schmittassociates.com
}

Received 12 October 2009

Accepted for publication 11 December 2009

Published 22 January 2010

Online at stacks.iop.org/JBR/4/026001

\begin{abstract}
Viral infections cause increased oxidative stress, so a breath test for oxidative stress biomarkers (alkanes and alkane derivatives) might provide a new tool for early diagnosis. We studied 33 normal healthy human subjects receiving scheduled treatment with live attenuated influenza vaccine (LAIV). Each subject was his or her own control, since they were studied on day 0 prior to vaccination, and then on days 2, 7 and 14 following vaccination. Breath volatile organic compounds (VOCs) were collected with a breath collection apparatus, then analyzed by automated thermal desorption with gas chromatography and mass spectroscopy. A Monte Carlo simulation technique identified non-random VOC biomarkers of infection based on their $C$-statistic values (area under curve of receiver operating characteristic). Treatment with LAIV was followed by non-random changes in the abundance of breath VOCs. 2, 8-Dimethylundecane and other alkane derivatives were observed on all days. Conservative multivariate models identified vaccinated subjects on day $2(C$-statistic $=0.82$, sensitivity $=63.6 \%$ and specificity $=88.5 \%)$; day $7(C$-statistic $=0.94$, sensitivity $=88.5 \%$ and specificity $=92.3 \%)$; and day $14(C$-statistic $=0.95$, sensitivity $=92.3 \%$ and specificity $=92.3 \%)$. The altered breath VOCs were not detected in live attenuated influenza vaccine, excluding artifactual contamination. LAIV vaccination in healthy humans elicited a prompt and sustained increase in breath biomarkers of oxidative stress. A breath test for these VOCs could potentially identify humans who are acutely infected with influenza, but who have not yet developed clinical symptoms or signs of disease.
\end{abstract}

(Some figures in this article are in colour only in the electronic version)

\section{Abbreviations \\ ATD \\ BCA automated thermal desorption \\ $\mathrm{GC}$ breath collection apparatus \\ ${ }^{6}$ Author to whom any correspondence should be addressed.}

\section{MS mass spectroscopy \\ VOCs volatile organic compounds}

Early diagnosis of influenza could result in improved medical management by decreasing the use of antibiotics, encouraging antiviral therapy, and aiding the containment of pandemic disease [1]. An ideal test would detect early infection with 
influenza prior to the onset of symptomatic disease, and it should be sensitive, specific, non-invasive and cost-effective. Breath testing for volatile organic compounds (VOCs) offers a potentially useful new approach to early diagnosis of viral infections, because infection with influenza viruses results in increased oxidative stress [2], which in turn may result in increased excretion of VOC biomarkers in the breath, including alkanes and methylated alkanes [3, 4].

Oxidative stress plays a key pathophysiological role in the pathogenesis of several viral diseases. The high output of nitric oxide (NO) from inducible NO synthase produces highly reactive nitrogen oxide species, such as peroxynitrite, via interaction with oxygen radicals and reactive oxygen intermediates. These reactive nitrogen species cause oxidative tissue injury and mutagenesis through oxidation and nitration of various biomolecules [5]. These mechanisms have been mainly elucidated in animal models. Kumar et al instilled influenza virus in mice and observed increased superoxide radical production and lipid peroxidation products by alveolar macrophages on the fifth day after virus instillation [6]. Since there is a lack of comparable studies in humans, we studied breath biomarkers of oxidative stress in human subjects undergoing vaccination with live attenuated influenza virus (LAIV).

The objectives of this study were first, to determine the effects of LAIV on the composition of volatile organic compounds (VOCs) in human breath, and second, to distinguish between breath VOCs elicited by human physiological responses to influenza vaccination and VOCs that may have comprised components of the vaccine.

\section{Methods}

\section{Experimental design}

Each subject was his or her own control, since they were studied on day 0 prior to vaccination, and then on days 2, 7 and 14 following vaccination. Breath VOC samples were collected by Air Force medical personnel at Eglin Air Force Base, FL and sent to the Breath Research Laboratory of Menssana Research, Inc., Newark, NJ, for analysis.

\section{Human studies}

We studied 33 normal healthy human subjects (mean age = 28.2 years, $\mathrm{SD}=6.5$; male $/$ female $=29 / 4$ ). All were undergoing scheduled treatment with LAIV in accordance with guidelines established by the Department of Defense and the Centers for Disease Control. All participants gave their written informed consent to participate in this study, which was approved by the Regional Institutional Review Board at the Wilford Hall USAF Medical Center, Lackland Air Force Base, TX.

\section{Influenza vaccination}

All subjects were treated with LAIV (FluMist ${ }^{\circledR}$ Influenza Virus Vaccine Live, Intranasal Spray, MedImmune, LLC, Gaithersburg, MD 20878). Approximately $0.1 \mathrm{~mL}$ (half of the dose from a single FluMist ${ }^{\circledR}$ sprayer) was administered into each nostril while the participant was in an upright position.

\section{Collection of breath VOC samples}

The method has been described [3]. In summary, subjects respired normally for $2.0 \mathrm{~min}$ through a disposable-valved mouthpiece and a bacterial filter into a portable breath collection apparatus (BCA), while wearing a nose clip. VOCs in $1.0 \mathrm{~L}$ alveolar breath and $1.0 \mathrm{~L}$ room air were captured on to separate sorbent traps.

\section{Analysis of breath VOCs}

The method has been described [3]. VOCs captured in the sorbent traps were analyzed in the laboratory by automated thermal desorption, gas chromatography and mass spectroscopy (ATD/GC/MS). In order to quantify peak areas and to control for drift in instrument performance, an internal standard was run with every chromatographic assay of breath and air $(0.25 \mathrm{~mL} 2 \mathrm{ppm}$ 1-Bromo-4-fluorobenzene, Supelco, Bellefonte, PA).

\section{Analysis of influenza virus vaccine VOCs}

The contents of six syringes of FluMist ${ }^{\circledR}$ Influenza Virus Vaccine Live $(0.2 \mathrm{~mL}$ dose/syringe. Exp. Date 31March08 Lot 500534P) were loaded into separate crimp-top vials and sealed. Two crimp-top vials were respectively equilibrated for $15 \mathrm{~min}$ at $0{ }^{\circ} \mathrm{C}$ on crushed ice, room temperature and at $37{ }^{\circ} \mathrm{C}$. Head space samples $(2.0 \mathrm{~mL})$ were withdrawn into a heated syringe, injected onto sorbent traps similar to those employed for collection of breath VOCs, and analyzed in the same fashion.

\section{Analysis of breath and air VOC data}

Chromatographic data. The alveolar gradient (abundance in breath minus abundance in ambient room air) of each VOC $\left(\mathrm{AG}_{\mathrm{VOC}}\right)$ was determined as follows. $\mathrm{AG}_{\mathrm{VOC}}=V_{\mathrm{b}} / I_{\mathrm{b}}-V_{\mathrm{a}} / I_{\mathrm{a}}$, where $V_{\mathrm{b}}$ was the area under the curve (AUC) of the VOC peak in the alveolar breath chromatogram, $I_{\mathrm{b}}$ was the AUC of the internal standard peak and $V_{\mathrm{a}}$ and $I_{\mathrm{a}}$ were corresponding values derived from the associated chromatogram of room air [7]. A similar quantity of internal standard (bromofluorobenzene) was injected with all assays of breath and air VOCs, so that the ratios of $V_{\mathrm{b}} / I_{\mathrm{b}}$ and $V_{\mathrm{a}} / I_{\mathrm{a}}$ varied with the molar concentrations of the VOCs in breath and in air.

Construction of multivariate algorithms. A Monte Carlo simulation was employed to select the breath VOCs that identified vaccination with better than random accuracy. The accuracy of a VOC as a biomarker of vaccination was initially estimated by comparing its alveolar gradient values in subjects before and after treatment with live attenuated influenza virus, and determining the value of its $C$-statistic, i.e. the AUC of the receiver operating characteristic (ROC) curve [8]. The average random behavior of breath VOCs was determined by randomly assigning subjects to the 'before treatment' or 'after 
treatment' groups, and performing multiple estimates $(n=$ 40) of each breath VOC's $C$-statistic value. For any given value of the $C$-statistic, it was then possible to compare the number of VOCs exceeding that value by correct assignment $\left(n_{1}\right)$ or by random assignment $\left(n_{2}\right)$, so that the better than random biomarkers of infection comprised a subset of $n_{1}-n_{2}$ correctly assigned VOCs. All combinations of $n_{1}-n_{2}$ assigned VOCs were combined in predictive algorithms employing weighted digital analysis (WDA), a multivariate analysis procedure [9] and the most conservative multivariate algorithm (i.e. the algorithm with the lowest $C$-statistic) was selected. The predictive algorithm with the true value was unknown, and its accuracy was probably higher than the most conservative algorithm with the lowest $C$-statistic value.

\section{Results}

\section{Human subjects}

None of the subjects reported any adverse effects, either of influenza vaccination or the breath collection procedure.

Breath biomarkers of treatment with live attenuated influenza virus. A representative Monte Carlo simulation using breath VOC $C$-statistics from day 14 of the study is shown in figure 1.

Mean outcomes of 40 Monte Carlo simulations on days 2, 7 and 14 following influenza vaccination are shown in figure 1 . The excess of correct over random VOCs $\left(n_{1} / n_{2}\right)$ was $12 / 5$ on day $2,7 / 6$ on day 7 and $3 / 0$ on day 14 .

ROC curves on days 2,7 and 14 following influenza vaccination are shown in figure 1 , employing the most conservative multivariate algorithm derived from $n_{1}-n_{2}$ VOCs. On day $2 C$-statistic $=0.82$, sensitivity $=63.6 \%$ and specificity $=88.5 \%$; day $7 C$-statistic $=0.94$, sensitivity $=$ $88.5 \%$ and specificity $=92.3 \%$ and day $14 C$-statistic $=0.95$, sensitivity $=92.3 \%$ and specificity $=92.3 \%$.

Breath biomarkers of vaccination. Tentative MS identifications of VOCs employed in algorithms on days 2, 7 and 14 following influenza vaccination are shown in table 1 .

\section{Analysis of influenza virus vaccine VOCs}

Tentative MS identifications of the top 20 most abundant VOCs observed in the headspace samples of FluMist ${ }^{\circledR}$ Influenza Virus Vaccine Live are shown in table 1.

\section{Discussion}

Treatment with LAIV was accompanied by sustained changes in the abundance of VOCs in breath. The combined changes in the abundance of these breath VOCs identified treated subjects at 2, 7 and 14 days after viral exposure. These findings were consistent with altered endogenous manufacture of VOCs as a physiological response by normal humans to a challenge with LAIV. Since the altered breath VOCs were not present in the head space of the vaccine, it is unlikely that these VOCs were contamination artifacts derived from the vaccine itself. The mean alveolar gradients of these VOCs were either increased or decreased compared to their baseline values prior to LAIV exposure.

Breath VOCs were tentatively identified by mass spectroscopy, and the set of apparent biomarkers varied during the course of the study, consistent with an evolving response to the viral challenge. 2,8-dimethyl-undecane was identified on all days, and other alkane derivatives were also observed, including 4,8-dimethyl-undecane and 2-isopropyl-5-methyl1-heptanol.

We hypothesize that infection with LAIV affected the composition of breath VOCs in two ways: first, by increased oxidative stress, and second by inhibition of cytochrome $\mathrm{p} 450$ activity (see figure 2). LAIV may have initiated oxidative stress by a similar mechanism observed in influenza virus pneumonia, with the production of highly reactive nitrogen oxide species, such as peroxynitrite, via interaction with oxygen radicals and reactive oxygen intermediates [5]. These highly reactive free radicals are powerful oxidizing agents due to unpaired electrons in their outer valence orbitals [10]. Influenza vaccination has also been previously reported as a powerful inhibitor of cytochrome p450 activity [16-18], which may account for the consistent and significant reduction observed in the mean alveolar gradient of 2,8-dimethylundecane as well as the reduced abundance of other VOCs (see table 1 and figure 2). The breath biomarker response was observed throughout the 14 days of the study, which may have been due to evolving host immune response with or without persistent viral replication. In mouse models of experimental influenza infection, the virus has been detected for similar periods following intranasal administration $[11,12]$.

A central problem in a biomarker discovery study is the need to distinguish true from false biomarkers of disease. When a large number of VOCs are assayed in a comparatively small number of subjects, some non-biomarker VOCs may appear to be accurate identifiers of a disease solely because of random statistical fluctuations. Monte Carlo simulation techniques provide a powerful mathematical tool for distinguishing between true and false biomarkers. Our Monte Carlo simulations addressed three questions: First, what was the $C$-statistic of a VOC when the diagnosis was assigned by chance alone? Second, what was the $C$-statistic of that VOC when the diagnosis was assigned correctly? Third, what was the difference between these two values?

In order to answer the first question, multiple simulations employing random assignment of diagnosis are performed in the same patient dataset. We performed 40 simulations in order to determine the mean and standard deviation of the $C$-statistic of each VOC using random assignment of diagnosis (i.e. preor post-exposure to LAIV). As shown in figure 1, Monte Carlo simulations demonstrated that for any given value of the $C$ statistic, a greater number of VOCs was observed employing the correct diagnosis than with random assignment. At the point where the random assignment curve fell to zero, the VOCs remaining in the correct diagnosis curve were the true biomarkers of disease. 

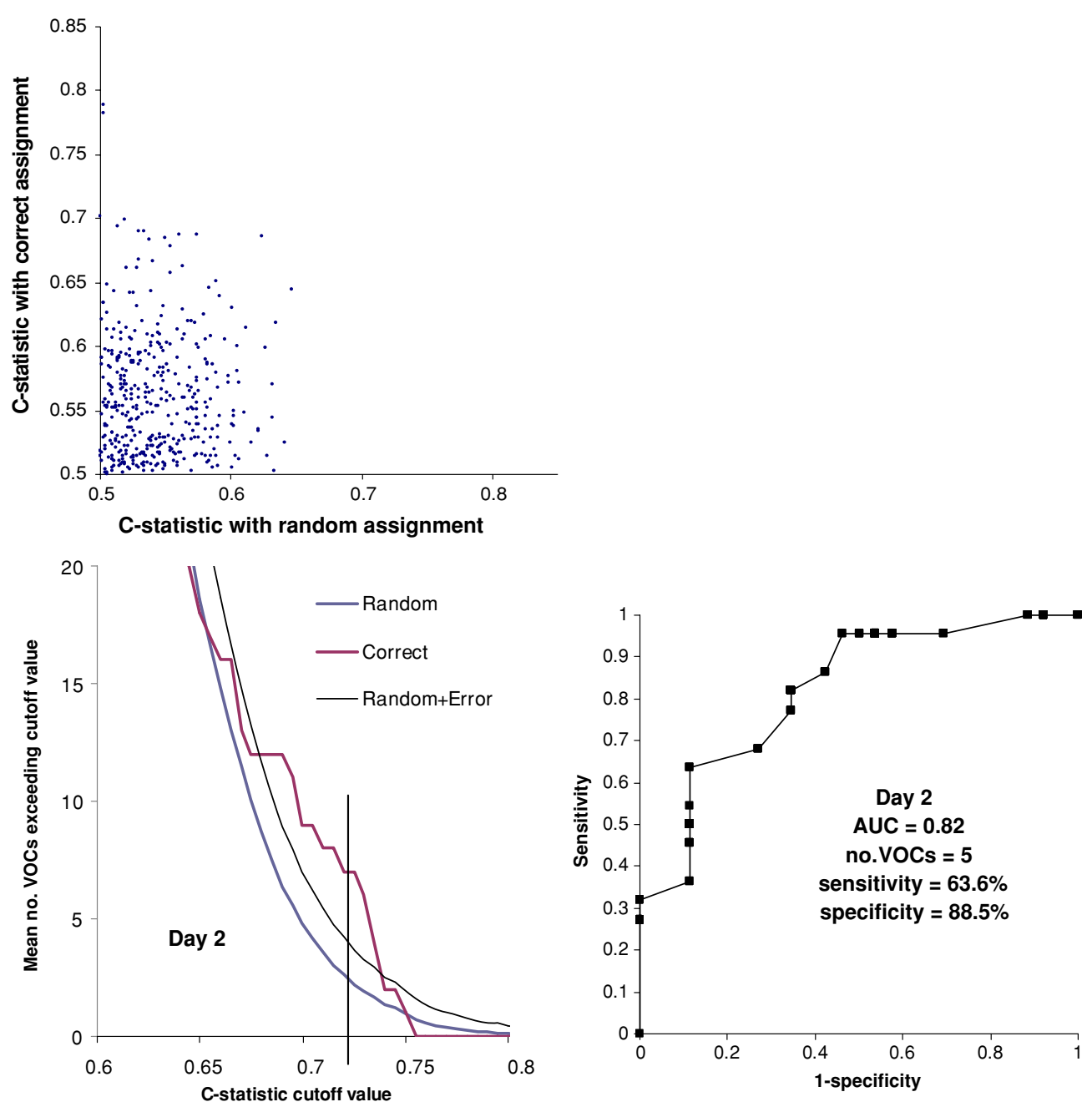

Figure 1. Monte Carlo simulations and ROC curves. Single Monte Carlo simulation (top left panel): each breath VOC was evaluated as a candidate biomarker of vaccination with live attenuated influenza virus by comparing its alveolar gradients in subjects before and after exposure. This figure displays the $C$-statistic of each breath VOC employing either randomized or correct assignment to viral exposure groups on the $x$ and $y$ axes respectively. In this particular simulation comparing breath VOCs on day 14 to pre-exposure levels; there is an excess of VOCs with $C$-statistic $>0.65$ compared to the results observed with randomized assignment. Outcome of multiple Monte Carlo simulations (bottom three left-hand panels): these display the mean outcome of 40 Monte Carlo simulations similar to the single simulation shown at top, on days 2, 7 and 14 following influenza vaccination. Curves display the mean number of VOCs exceeding a given AUC cutoff with random and correct assignment to the vaccination group. The random + error curve displays the mean random curve plus 1.0 SD. If a breath VOC signal of vaccination is present, the correct assignment curve should be significantly higher than the randomized curve. The difference between the correct and randomized curve varies with the number of true biomarkers present in the data. On day 2, at $C$-statistic cutoff value $=0.72$, a mean of seven VOCs was observed with correct assignment of viral exposure and two VOCs with randomized assignment. The excess of correct over random VOCs indicated that five VOCs comprised non-random biomarkers of exposure to attenuated influenza virus. There was a similar excess of correct over random VOCs on day $7(6 / 0$ at $C$-statistic cutoff value $=0.74)$ and on day 14 (3/0 at $C$-statistic cutoff value $=0.75)$. Receiver operating characteristic $(R O C)$ curves (bottom three right-hand panels): for a given value of the $C$-statistic, $n_{1}$ VOCs exceeded that value by correct assignment and $n_{2}$ by random assignment, so that the better than random biomarkers of infection comprised a subset of $n_{1}-n_{2}$ correctly assigned VOCs. All combinations of $n_{1}-n_{2}$ correctly assigned VOCs were combined in predictive algorithms employing weighted digital analysis (WDA), a multivariate analysis procedure. ROC curves are shown that employed the most conservative multivariate algorithm (i.e. the algorithm with the lowest $C$-statistic). The number of VOCs selected for each multivariate model is shown.

These Monte Carlo simulations enabled us to select a set of breath biomarker VOCs based on their individual $C$ statistic values, and to determine the lower limit of the accuracy of the multivariate WDA model. Monte Carlo methods are computational algorithms that achieve their results by using repeated random sampling. Originally developed to simulate physical and mathematical systems, the term was coined in the 1940s by physicists working on nuclear weapon projects in the Los Alamos National Laboratory [13]. Monte Carlo simulations have been increasingly employed in recent years for biological applications such as the identification of biomarkers [14, 15].

WDA is a multivariate analysis technique that simulates the reasoning that doctors employ to diagnose a disease. 

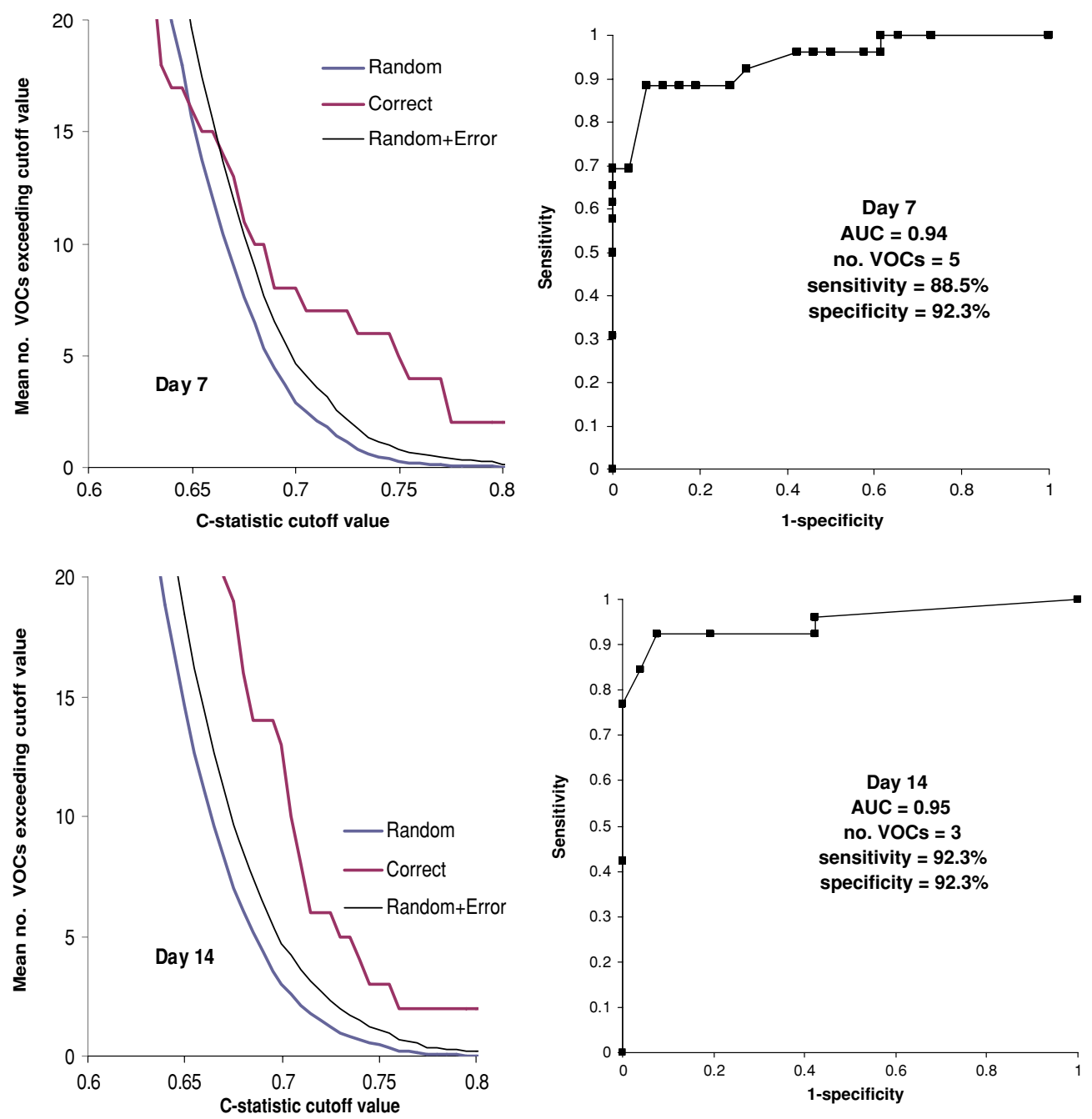

Figure 1. (Continued.)

Physicians may count the number of symptoms or signs present in a patient that are consistent with a particular disease (e.g. the Jones criteria for rheumatic fever), and the diagnosis becomes positive if the number of symptoms or signs exceeds a threshold value. WDA generates a discriminant function to predict membership in one of two groups (in this case, disease versus no disease) by determining three parameters for each predictor variable employed in the model: a weight, a cutoff value, and a sign. The weight of each predictor variable is the AUC of the ROC curve minus a fixed offset of 0.55 , where the AUC is obtained by employing that particular predictor variable as the sole marker of disease. The cutoff value is determined as the point on the ROC curve where the sum of sensitivity plus specificity is maximal. The sign (plus or minus) is used to invert the predictor variable if a lower value indicated a higher probability of disease.

The role of breath testing for influenza has not yet been established, but rapid screening in a physician's office might provide an appropriate clinical niche.

Although the clinical value of this test is not yet known, its potential value may be estimated by comparison to rapid screening tests in current use. The Centers for Disease Control and Prevention provide a guide to 'Influenza Symptoms and Laboratory Diagnostic Procedures' which states that serological testing for influenza requires paired acute and convalescent sera, and does not provide results that help with clinical decision making. Using respiratory samples (e.g. a nasopharyngeal or nasal swab) collected within the first 4 days of illness, most of the rapid tests that can be done in a physician's office are approximately $50-70 \%$ sensitive for detecting influenza and $90 \%$ specific. In comparison, the breath test for LAIV achieved $82 \%$ accuracy on day 2, with sensitivity $=63.6 \%$ and specificity $=88.5 \%$, rising to $94 \%$ accuracy on day 7 , with sensitivity $=88.5 \%$ and specificity $=92.3 \%$. These findings suggest that a breath test would be at least as accurate as rapid screening tests in current use, and possibly superior.

This study employed benchtop GC/MS instruments, so that breath VOC assays were comparatively slow and expensive. However, the VOCs identified during the feasibility phase of a clinical study might be identified in the future with point-of-care (POC) instruments that are optimized to 
Table 1. Identification of VOCs in breath and in influenza virus vaccine.

Panel A: chemical structure of breath biomarkers following exposure to live attenuated influenza virus ${ }^{\mathrm{a}}$

\begin{tabular}{llll}
\hline & $C$-statistic & CAS number & Change \\
\hline Day 2 & & & \\
Benzene, 1-methyl-4-(1-methylethenyl)- & 0.75 & $1195-32-0$ & - \\
4-Penten-2-ol & 0.74 & $625-31-0$ & + \\
2,5-Cyclohexadiene-1,4-dione, 2,6-bis(1,1-dimethylethyl)- & 0.73 & $719-22-2$ & + \\
Undecane, 2,8-dimethyl- & 0.73 & $17301-25-6$ & - \\
1H-Indene, 1-methylene- & 0.73 & $2471-84-3$ & + \\
á-Myrcene & 0.73 & $1686-30-2$ & + \\
o-Isopropenyltoluene & 0.72 & $7399-49-7$ & - \\
& & & \\
Day 7 & & & \\
Undecane, 2,8-dimethyl- & 0.84 & $17301-25-6$ & - \\
Undecane, 4,8-dimethyl- & 0.81 & $17301-33-6$ & - \\
2-Isopropyl-5-methyl-1-heptanol & 0.77 & $91337-07-4$ & - \\
1H-indene, 1-methylene- & 0.77 & $2471-84-3$ & + \\
Azulene & 0.75 & $275-51-4$ & + \\
& & & \\
Day 14 & & & \\
Undecane, 2,8-dimethyl- & 0.85 & Not available & + \\
(7a-Isopropenyl-4,5-dimethyloctahydroinden-4-yl)methanol & 0.82 & $874-41-9$ & + \\
Benzene, 1-ethyl-2,4-dimethyl- & 0.75 & \\
\hline
\end{tabular}

Panel B: chemical structure of the VOCs observed in the head space samples of FluMist ${ }^{\circledR}$ Influenza Virus Vaccine Live ${ }^{\mathrm{b}}$

\begin{tabular}{ll}
\hline Compounds in head space of influenza vaccine & CAS number \\
\hline Benzonitrile & $100-47-0$ \\
Tricyclo[3.1.0.0[2,4]]hex-3-ene-3-carbonitrile & $103495-51-8$ \\
Isopropyl Palmitate & $142-91-6$ \\
N-Cbz-glycylglycine p-nitrophenyl ester & $13574-81-7$ \\
n-Hexadecanoic acid & $57-10-3$ \\
1,1-Biphenyl, 2-methyl- & $643-58-3$ \\
Cyclohexane, 1,1-dodecylidenebis[4-methyl- & $55334-09-3$ \\
Benzene, 1-methyl-4-[phenylmethyl]- & $620-83-7$ \\
Diphenylmethane & $101-81-5$ \\
Heptadecane, 9-octyl- & $7225-64-1$ \\
Heptadecane, 2,6-dimethyl- & $54105-67-8$ \\
tert-Hexadecanethiol & $25360-09-02$ \\
Heptadecane, 2,6,10,15-tetramethyl- & $54833-48-6$ \\
Vinylfuran & $1487-18-9$ \\
Phenol & $13127-88-3$ \\
10,18-Bisnorabieta-8,11,13-triene & $32624-67-2$ \\
Malonic acid, bis[2-trimethylsilylethyl ester & $90744-45-9$ \\
Ethanedioic acid, bis[trimethylsilyl] ester & $18294-04-7$ \\
Phosphonic acid, [p-hydroxyphenyl]- & $33795-18-5$ \\
\hline
\end{tabular}

a The VOCs employed in the ROC curves on days 2, 7 and 14 are shown, with their individual $C$-statistic values and CAS numbers. 'Change' indicates whether the mean alveolar gradient increased $(+)$ or decreased (-) compared to its baseline value prior LAIV exposure. Chemical structures were tentatively identified by mass spectroscopy. These VOCs appear to represent physiological responses to vaccination, and not components of the vaccine, because none of these VOCs are common to the list of VOCs observed in the head space samples of FluMist ${ }^{\circledR}$ Influenza Virus shown in panel B. On day 2, five of the seven VOCs were true markers and two were derived from random fluctuations; it is not possible to determine which five were the 'true' markers.

${ }^{b}$ The 20 most abundant VOCs are shown, ranked from the most abundant VOC at the head of the list.

detect disease biomarkers rapidly and at lower cost. In clinical practice, non-invasive and inexpensive POC instruments might enable sequential breath tests in healthy people prior to and then during influenza season. This could establish the baseline abundance of biomarkers in an individual, so that a breath test might reveal acute infection during the incubation phase of influenza before symptoms and signs of disease develop.
These findings demonstrate that receipt of LAIV in healthy humans was accompanied by statistically significant changes in the pattern of breath VOCs. It is likely that infected humans exhaled oxidative stress products and other VOCs in their breath in an apparent physiological response to treatment with LAIV. These findings suggest that breath VOC biomarkers of oxidative stress could potentially identify 


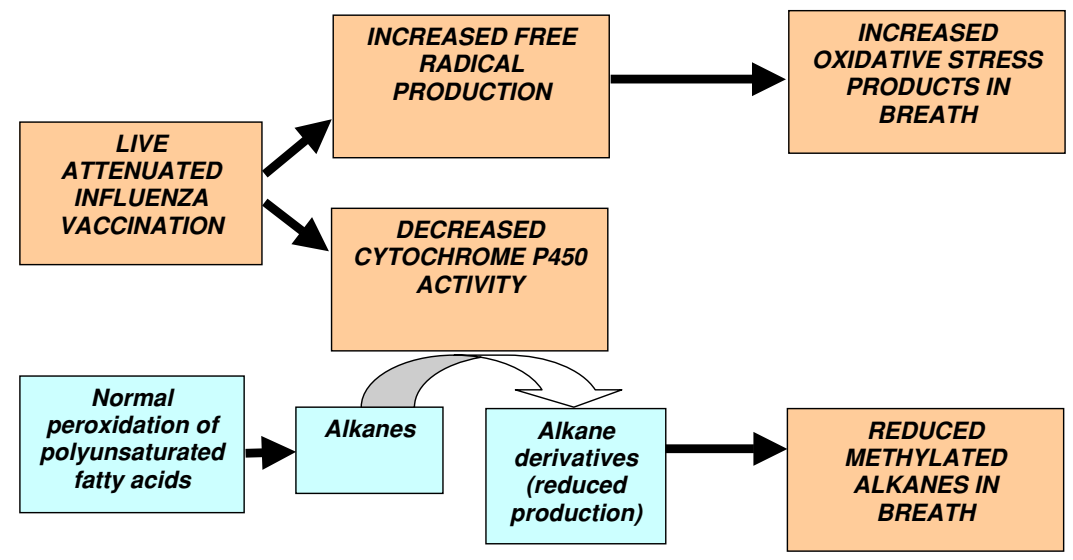

2,8-dimethylundecane

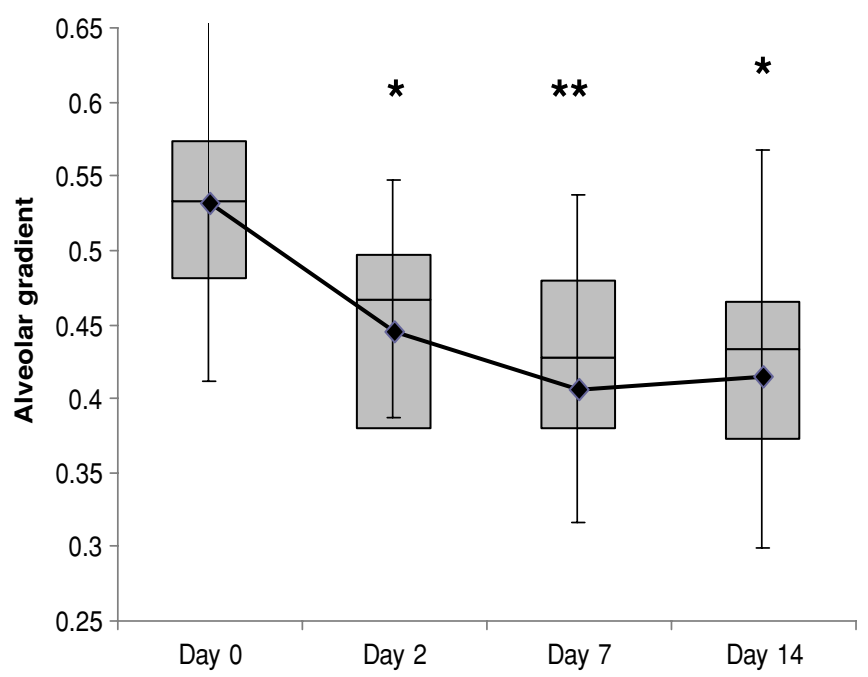

Figure 2. Breath test response to viral infection. Upper panel: hypothetical mechanism. Infection with LAIV initiates two processes: the production of highly reactive free radicals, and the inhibition of cytochrome p450 activity. Free radicals are powerful oxidizing agents, due to unpaired electrons in their outer valence orbitals. The resulting oxidative stress increases the abundance of volatile oxidized metabolites in the breath. Inhibition of cytochrome p450 activity reduces the conversion of normal metabolites such as alkanes to derivatized VOCs, possibly accounting for the reduced abundance of 2,8-dimethylundecane and other alkane products in the breath. Lower panel:

2,8-dimethylundecane in breath. The figure displays the mean alveolar gradient of 2,8-dimethylundecane in 33 normal healthy human subjects over 14 days following LAIV exposure, with 25 th, 50th and 75 th percentiles, and standard deviation. ${ }^{*} p \leqslant 0.01,{ }^{* *} p \leqslant 0.001$. The mean alveolar gradient declined significantly, consistent with reduced synthesis of 2,8-dimethylundecane resulting from inhibition of cytochrome p450 activity.

humans who are acutely infected with influenza, but who have not yet developed clinical symptoms or signs of disease. Further studies are needed to confirm this possibility.

\section{Acknowledgments}

This research was supported by US Air Force Battlelab award FA3047-06-P-1166. Michael Phillips is President and CEO of Menssana Research, Inc. All coauthors had full access to all of the data in the study and they take responsibility for the integrity of the data and the accuracy of the data analysis. We thank James J Grady, DrPH, for statistical advice during the preparation of this report.

\section{References}

[1] Noyola D E and Demmler G J 2000 Effect of rapid diagnosis on management of influenza A infections Pediatr. Infect. Dis. J. 19 303-7

[2] Schwarz K B 1996 Oxidative stress during viral infection: a review Free Radic. Biol. Med. 21 641-9

[3] Phillips M, Cataneo R N, Greenberg J, Gunawardena R, Naidu A and Rahbari-Oskoui F 2000 Effect of age on the breath methylated alkane contour, a display of apparent new markers of oxidative stress J. Lab. Clin. Med. 136 243-9

[4] Phillips M, Cataneo R, Greenberg J, Grodman R, Gunawardena R and Naidu A 2003 Effects of oxygen on breath markers of oxidative stress Eur. Respir. J. 21 48-51

[5] Akaike T 2001 Role of free radicals in viral pathogenesis and mutation Rev. Med. Virol. 11 87-101

[6] Kumar P, Sharma S, Khanna M and Raj H G 2003 Effect of Quercetin on lipid peroxidation and changes in lung 
morphology in experimental influenza virus infection Int. J. Exp. Pathol. 84 127-33

[7] Phillips M 1997 Method for the collection and assay of volatile organic compounds in breath Anal. Biochem. 247 272-8

[8] Cook N R 2007 Use and misuse of the receiver operating characteristic curve in risk prediction Circulation $115928-35$

[9] Phillips M et al 2008 Detection of lung cancer using weighted digital analysis of breath biomarkers Clin. Chim. Acta; $39376-84$

[10] Boveris A 1998 Biochemistry of free radicals: from electrons to tissues Medicina 58 350-6

[11] Davis L E, Kornfeld M, Daniels R S and Skehel J J 2000 Experimental influenza causes a non-permissive viral infection of brain, liver and muscle J. Neurovirol. 6 529-36

[12] Mori I, Komatsu T, Takeuchi K, Nakakuki K, Sudo M and Kimura Y 1995 Viremia induced by influenza virus Microb. Pathog. 19 237-44
[13] Metropolis N and Ulam S 1949 The Monte Carlo method J. Am. Stat. Assoc. 44 335-41

[14] Ma S, Song X and Huang J 2006 Regularized binormal ROC method in disease classification using microarray data $B M C$ Bioinformatics 7253

[15] Zou Y Y, Yang J and Zhu J 2006 A robust statistical procedure to discover expression biomarkers using microarray genomic expression data J. Zhejiang Univ. Sci. 7 603-7

[16] Upton R A 1991 Pharmacokinetic interactions between theophylline and other medication (Part I) Clin. Pharmacokinet. 20 66-80

[17] Hayney M S and Buck J M 2002 Effect of age and degree of immune activation on cytochrome P450 3A4 activity after influenza immunization Pharmacotherapy 22 1235-8

[18] Lipsky B A, Pecoraro R E, Roben N J, de Blaquiere P and Delaney C J 1984 Influenza vaccination and warfarin anticoagulation Ann. Intern. Med. 100 835-7 